\title{
Neurochirurgie - aktuelle und zukünftige Konzepte einer verbesserten operativen Therapie
}

\author{
G. Schackert R. Steinmeier \\ Klinik und Poliklinik für Neurochirurgie, TU Dresden
}

\section{Einleitung}

Die operativen Verfahren in der Neurochirurgie wurden in den letzten Jahren wesentlich von technischen Neuerungen und computergestützten Methoden beeinflusst. Während der Beginn hirnchirurgischer Eingriffe auf Grund mangelnder diagnostischer, anästhesiologischer und operativer Verfahren von hoher Mortalität und Morbidität gekennzeichnet war, konnte durch die Fortschritte in der modernen Technik ein hoher Sicherheitsstandard erreicht werden.

Das Ziel neurochirurgischer Eingriffe ist die Erhaltung der Hirnfunktionen und die möglichst vollständige Entfernung der pathologischen Strukturen. Durch die moderne diagnostische Bildgebung wie die Magnetresonanztomographie (MRT), die Computertomographie und die Angiographie zur Visualisierung morphologischer Strukturen sowie die Positronen-Emissionstomographie (PET) oder die MR-Spektroskopie (MR-SPECT) zur Darstellung des Stoffwechsels gelingt es, krankhafte Veränderungen frühzeitig zu erkennen und mit geeigneten Operationsverfahren anzugehen. In zahlreichen klinischen Studien konnte gezeigt werden, dass einer der wesentlichen Parameter, der die Prognose des Patienten signifikant beeinflusst, der klinische Zustand und damit der «Karnofsky Performance Score» (KPS) ist. Aktuelle und zukünftige Operationskonzepte streben daher eine weitere Verbesserung der Funktionserkennung unter routinemäßiger Einbindung bildgebender Modalitäten an, die in den Operationssitus projiziert werden. Im Folgenden wird anhand von Beispielen aus der Tumorchirurgie auf aktuelle Verfahren und deren Entwicklungsmöglichkeiten eingegangen werden.

\section{Gliomchirurgie}

Gliome bilden etwa $30-40 \%$ der Hirntumoren. Sie stellen damit die größte Gruppe unter den Hirntumoren dar. Zu ihnen zählen die Astrozytome, Oligodendrogliome, Ependy- mome und die Tumoren des Plexus choroideus. Die häufigsten Gliome sind die Astrozytome. An ihrem Beispiel sollen die operativen Vorgehensweisen näher erklärt werden. Die Astrozytome werden nach der WHO in vier Dignitätsgrade eingeteilt. Dies ist von Bedeutung für die operative Vorgehensweise (Tab. 1).

Das Charakteristikum der Gliome ist ihr infiltratives Wachstum und der Mangel an einer Tumorgrenze. Die Infiltrationsneigung steigt mit zunehmendem Malignitätsgrad. In vergleichenden Untersuchungen an Computertomographie- und Autopsieschnitten konnte gezeigt werden, dass bei Glioblastomen, d.h. den Grad-IV-Gliomen, noch in mehreren Zentimetern Abstand von der sich bildgebend anreichernden Tumorgrenze Tumorzellen nachgewiesen werden können [1]. Damit wird deutlich, dass Gliome operativ nicht kurativ zu entfernen sind, und immer in der peritumoralen Zone Tumorzellen im Normalgewebe zurückbleiben.

\section{Methoden zur Erhaltung der Funktionalität}

Wie aus dem oben Gesagten hervorgeht, ist die Erhaltung der Hirnfunktion oberstes Ziel neurochirurgischer Therapiekonzepte. In den letzten Jahren wurden Verfahren entwickelt, die auf der einen Seite die Funktionserkennung und den Funktionserhalt und auf der anderen Seite eine möglichst vollständige Tumorresektion anstreben (Tab. 2 und 3):

\section{Aktueller Stand}

Neuronavigation

Eine der wichtigsten Neuerungen in der Neurochirurgie ist die Einführung der Neuronavigation. Diese steht nun seit etwa 15 Jahren zur Verfügung. Bei diesem Verfahren handelt es sich um eine computergestützte Methode, mit der es möglich ist, 3D-Bilddatensätze («virtuelle Welt») mit dem OP-Situs («physikalische Welt») zu fusionieren («registrieren») und so eine räumliche Vorstellung von der Tumorlokalisation zu er-

\begin{tabular}{ll}
\hline KARGER & ( 2002 S. Karger GmbH, Freiburg \\
$\begin{array}{l}\text { Fax +497614520714 } \\
\begin{array}{l}\text { E-mail Information@Karger.de } \\
\text { www.karger.com }\end{array}\end{array}$ & Accessible online at: \\
www.karger.com/journals/onk
\end{tabular}

Prof. Dr. Gabriele Schackert Universitätsklinikum «Carl Gustav Carus» der Technischen Universität Dresden Fetscherstr. 74

D-01307 Dresden (Deutschland) 
Tab. 1. Dignitätsgrade der Astrozytome (WHO)

Grad I pilozytisches Astrozytom

Grad II fibrilläres, protoplasmatisches, gemistozytisches Astrozytom

Grad III anaplastisches Astrozytom

Grad IV Glioblastom

Tab. 2. Funktionserkennung

Darstellung der Sprachregion durch «Brain mapping»

Darstellung des Motorcortex durch Phasenumkehr

Sprach-PET

fMRT

Darstellung der Pyramidenbahn durch diffusionsgewichtetes MRT

Funktionelle Bildgebung, Image fusion und Integration in die

Neuronavigation («funktionelle Neuronavigation»)

halten. Gleichzeitig können mit diesen Daten nicht nur die Tumoren dargestellt werden, sondern auch die Haut, die Schädelkalotte, das Gehirn, die Ventrikel, die Sinus. Damit wird der Tumor in seiner Beziehung zu den ihn umgebenden Strukturen visualisiert. Dies ermöglicht, gezielte Trepanationen vorzunehmen und bei tiefgelegenen Prozessen die Rindeninzision so zu gestalten, dass durch den Zugangsweg funktionell wichtige Hirnareale geschont und keine zusätzlichen Ausfälle hervorgerufen werden. Der wesentliche Vorteil der Neuronavigation besteht darin, dass kleine, tiefgelegene und multiple Prozesse sicher aufgefunden werden und multiple Läsionen in einer operativen Sitzung entfernt werden können. Die Neuronavigation stellt damit eine Methode dar, die bei allen Hirnprozessen zur Lokalisierung eingesetzt werden kann, die aber auf Grund des «Brain shifts» keine Aussage zur Radikalität der Exstirpation zulässt [2]. Unter dem Brain shift versteht man die Verlagerung von Hirnstrukturen, die sich nach Liquorverlust und Tumorreduktion ergibt. Da die eingespielten Computerdaten auf präoperativem Imaging beruhen und nur die Haut bzw. Schädelkalotte registriert werden können, wird intraoperativ nach partieller Tumorexstirpation keine «real time» Information vermittelt, d.h. der aktuelle Stand kann nicht dargestellt werden.

\section{«Brain mapping» der Sprachregion}

Im Vordergrund des funktionellen Operierens steht die sichere Identifizierung funktionell eloquenter Hirnareale. Vordringlich sind dabei die Erkennung der Sprachregion mit Broca- und Wernickezentrum und die Darstellung des motorischen Cortex. Es ist heute bekannt, dass die räumliche Verteilung der Sprachregionen individuellen Schwankungen unterliegt [3] und zudem durch raumforderndes Tumorwachstum verlagert wird. Unter elektrophysiologischer Stimulation kann am wachen Patienten intraoperativ die Sprachregion exakt lokalisiert werden. Dieses Verfahren nennt man «Brain mapping» $[4,5]$. Dabei werden dem nicht-narkotisierten Patien- ten Bilder gezeigt, die er, während eine elektrische Stimulation der Hirnoberfläche im Bereich der Sprachregion erfolgt, benennen muss. Bei direkter Stimulation der Sprachregion kommt es zu einem reversiblen Funktionsausfall von Neuronen, der zu «speech disturbance» bzw. «speech arrest» führt [3]. Nach dem Konzept von Ojemann [3] kann mit dieser Methode auch zwischen essentiellen und nur an der Sprachproduktion beteiligten Regionen unterschieden werden, was hinsichtlich der Resektionsradikalität von neurochirurgischer Relevanz ist. Der Nachteil dieses Verfahrens besteht jedoch darin, dass der Patient während der Operation nicht in Vollnarkose ist, über Stunden ruhig liegen muss und damit einer großen psychischen Belastung ausgesetzt ist.

\section{Monitoring des Motorcortex}

Die Identifikation des Motorcortex ist dagegen ein vergleichsweise einfaches Verfahren, das routinemäßig angewandt wird und kaum Zeit in Anspruch nimmt. Der motorische Cortex wird unter Ableitung der somatosensorisch evozierten Potentiale (SSEP) am narkotisierten Patienten erkannt. Zwischen Gyrus postzentralis und G. präzentralis schlägt die Welle N 20 in P 20 um. Damit kann der Motorcortex eindeutig lokalisiert werden [6].

\section{Zukünftige Konzepte}

Zukünftige Brain-mapping-Verfahren haben zum Ziel, bereits präoperativ eine sichere Identifizierung der Sprachregion zu erreichen. Voraussetzung hierzu ist jedoch eine mathematische Quantifizierung des Brain shifts mit Bestimmung der Vektor-Größen aller beteiligten und verlagerten Hirnregionen. Momentan ist dies nur an Super-Computern mit mehrstündiger Rechenzeit realisierbar. Dazu sind zwei Vorgehensweisen möglich: zum einen das Sprach-Positronen-Emissionstomogramm (Sprach-PET), zum anderen das funktionelle Magnetresonanztomogramm (fMRT).

Sprach-PET mit ${ }^{18}$ F-FDG oder radioaktiv markiertem Wasser Durch Verabreichung von radioaktiv markierter ${ }^{18}$ Fluor-Desoxy-Glukose ( $\left.{ }^{18} \mathrm{~F}-\mathrm{FDG}\right)$ ist es möglich, den regionalen Glukosemetabolismus zu messen. Zum Nachweis spracheloquenter Areale können unterschiedliche Paradigmen eingesetzt werden, wobei sich das «Verben generieren» als relativ zuverlässig und robust erwiesen hat. Die Aktivierung der Hirnregion führt zu einer Zunahme des lokalen Glukosemetabolismus im Vergleich zum Ruhe-PET (Abb. 1).

Bei Anwendung von radioaktiv markiertem Wasserstoff wird die zerebrale Durchblutung gemessen, die in der Aktivierung deutlich erhöht ist. Der Vorteil dieses Verfahrens liegt in der besseren Auflösung, der Nachteil in der sehr kurzen Halbwertszeit des Nuklids.

\section{Funktionelle Magnetresonanztomographie (fMRT)}

Bei der fMRT wird die paramagnetische Aktivität von Deoxyhämoglobin im Vergleich zur diamagnetischen Aktivität von 


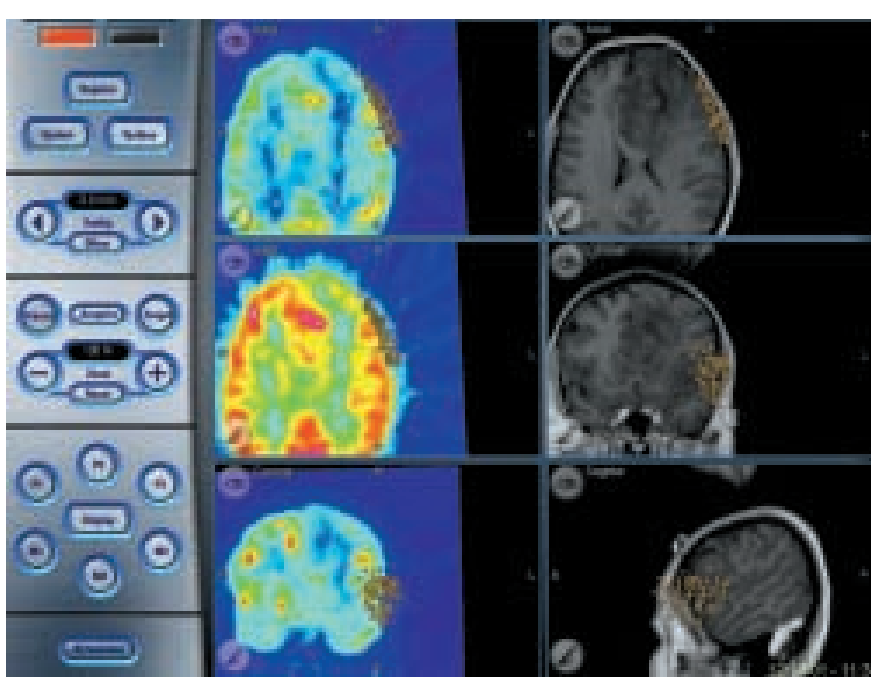

Abb. 1. Funktionelle Neuronavigation mit MRT und FDG-PET bei einer Patientin mit Astrozytom (WHO III). Bei der Operation wurde ebenfalls ein Brain mapping durchgeführt: Die Stimulationsorte sind im Bilddatensatz mit Zahlen markiert.
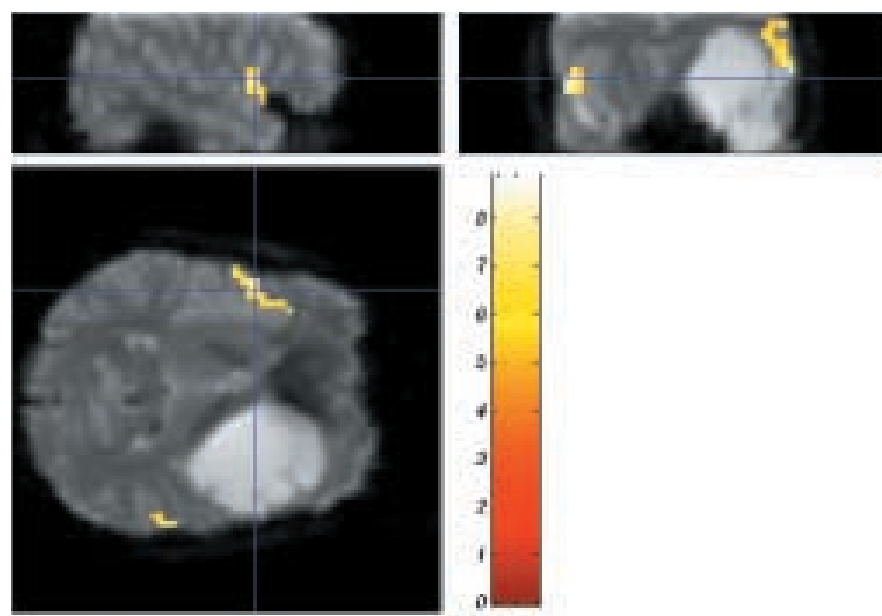

Abb. 2. fMRT der Sprachregion bei einer Patientin mit großem Gliom links fronto-temporal und Inselbeteiligung. Neben einer Aktivierung im Broka-Zentrum ist ebenfalls eine Aktivierung in der kontralateralen Inselregion zu erkennen.

Oxyhämoglobin genutzt. Unter kortikaler Aktivierung erhöht sich die Perfusion und damit nimmt auch der Anteil des oxygenierten Blutes und des Gewebe- $\mathrm{pO}_{2}$ zu. In GradientenEcho-Sequenzen (FLASH, EPI) kann das geänderte magnetische Grundfeld nach Signalmittelung und komplexer statistischer Auswertung bildlich dargestellt werden (Abb. 2). Eine standardisierte Bildauswertung existiert bisher nicht. Einzelnen Arbeitsgruppen ist jedoch eine räumliche Zuordnung des sensomotorischen Cortex, der primären Sehrinde oder der Sprachareale gelungen.

\section{Darstellung der Pyramidenbahn}

Jüngste Veröffentlichungen befassen sich mit der Darstellung der Pyramidenbahn zur Operationsplanung [7]. Damit wird

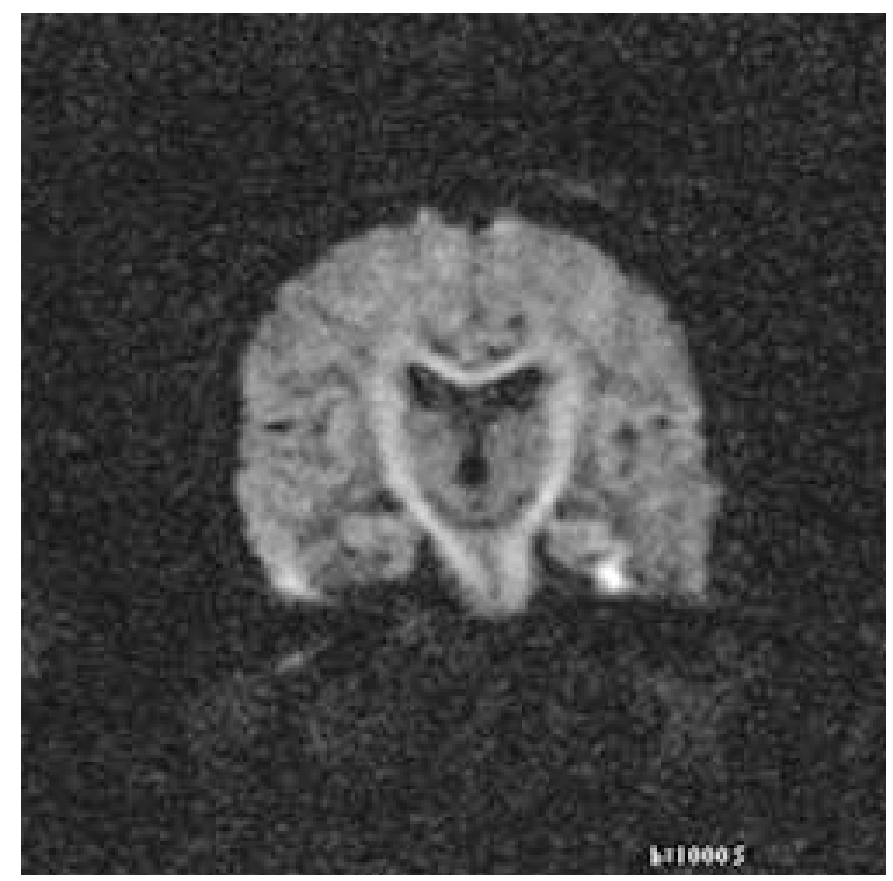

Abb. 3. Darstellung der Pyramidenbahn im diffusionsgewichteten MRT.

nicht wie bisher nur die oberflächliche Repräsentation der motorischen Region identifiziert, sondern es werden erstmals die motorischen Bahnen in den tiefen Hirnstrukturen wiedergegeben. In der diffusionsgewichteten Magnetresonanzuntersuchung (Abb. 3) bzw. dem «diffusion tensor imaging» ist über die durch den Faserverlauf der Bahnsysteme limitierte Flüssigkeitsdiffusion eine bildgebende Darstellung möglich. Die Untersuchungen wurden in einem 1,5-Tesla-Gerät mit speziellen Echosequenzen durchgeführt. Durch die Einbindung der MRT-Daten in das Navigationssystem gelingt eine dreidimensionale Wiedergabe und die Identifizierung intraoperativ zu Beginn der Operation. Zusätzlich kann durch die intraoperative Magnetstimulationen der motorisch evozierten Potentiale (MEPs) das Bahnsystem in real time detektiert werden [8].

\section{Image Fusion - funktionelle Neuronavigation}

Wie bereits oben ausgeführt stellt die Entwicklung der Neuronavigation, die über ein computergestütztes Operieren die sichere Orientierung im Schädelinneren und damit im Gehirn ermöglicht, den zentralen Fortschritt der letzten Jahre im neurochirurgischen Operationssaal dar. Zahlreiche Untersuchungen befassten sich in der Vergangenheit mit der Genauigkeit des Systems $[9,10]$ und dem Einfluss des Brain shifts auf die intraoperative Reliabilität der Daten. Weiterentwicklungen dieses Systems mit Verknüpfung von z.B. MRT-Daten mit PET-Untersuchungen oder MR-Angiographieerhebungen erlauben nicht nur morphologische Wiedergaben, sondern Lokalisierungen von Hirnfunktionen zur Operationsplanung. Die komplexe Einspielung von morphologischen und funktionellen Daten, die über eine Fusion unterschiedlicher Bildgebungen verwirklicht wird, stellt das Thema zukünftiger Thera- 
Tab. 3. Methoden zur maximalen Tumorresektion

Intraoperative Magnetresonanztomographie

Intraoperativer Ultraschall

Fluoreszenzgestützte Detektion von Tumorzellen

piekonzepte dar. PET-Daten oder fMRT-Untersuchungen zur Lokalisation der Sprachregion, die mit MRT-Bildgebung in Übereinstimmung gebracht werden, könnten das intraoperative Sprachmonitoring am wachen Patienten überflüssig machen. Die Wiedergabe der Pyramidenbahn mit Einbindung in das Navigations-MRT wird in zukünftigen Untersuchungen auf ihre Wertigkeit hinsichtlich der Sicherheit im Funktionserhalt geprüft werden müssen. Ein Problem wird der Brain shift bleiben, der nach Liquorabfluss und Tumorteilexstirpation entsteht. Aufwendige Rechenoperationen mit Bestimmung der Vektor-Größen der beteiligten Hirnregionen könnten zur Lösung dieses Problems beitragen. Dies muss das Ziel weiterer Forschungsprojekte sein.

\section{Methoden zur maximalen Tumorresektion}

Zur intraoperativen Sicherung der maximalen Tumorexstirpation stehen neben dem intraoperativen MRT und dem diagnostischen Ultraschall experimentelle Ansätze wie die Fluoreszenzdetektion von Glioblastomzellen zur Verfügung (Tab. 3).

\section{Aktueller Stand}

\section{Intraoperative Magnetresonanztomographie}

Die intraoperative Magnetresonanztomographie steht auf Grund des hohen finanziellen Aufwandes nur wenigen Kliniken zur Verfügung. Es gibt in Deutschland zur Zeit zwei Systeme, beide sind Niederfeld-Geräte (0,2 bzw. 0,5 Tesla). Bei dem 0,5-Tesla-Gerät handelt es sich um einen Tomographen, der in der Regel neben dem Operationssaal untergebracht ist. Zur Durchführung der intraoperativen Kontrolle muss der Patient aus dem Operationssaal in den benachbarten Raum transportiert werden, dies ist mit einer Operationszeitverlängerung verbunden $[11,12]$. Bei dem zweiten System handelt es sich um einen offenen 0,2 Tesla Magnetresonanztomographen, in dem die Operationen vorgenommen werden. Dies bedeutet eine vollständige magnetische Abschirmung des Operationssaales und eine amagnetische Ausstattung des Operationsinstrumentariums und des Operationsmikroskopes. Die ausgiebigsten Erfahrungen bestehen im Brigham and Women's Hospital in Boston [13]. Durch das MRT ist es möglich, intraoperativ zum Abschluss der Tumorexstirpation nach Kontrastmittelapplikation (Gadolinium) das Ausmaß der Resektion zu beurteilen. Von besonderer Bedeutung ist die intraoperative Resektionskontrolle bei niedergradigen Gliomen. Durch das infiltrative intraaxiale Wachstum der Tumoren ist es optisch oft nicht möglich, die Tumorgrenzen zu erkennen. Zudem erschwert die peritumorale Ödemzone die Unterscheidung von Tumor und Normalgewebe. Bei höhergradigen Gliomen (Grad III und IV) kann auch durch eine bildgebend radikale Tumorexstirpation das Rezidiv nicht verhindert werden. Dennoch scheint die weitestgehende Zytoreduktion die Zeit bis zum Auftreten des Rezidiv zu verlängern und damit die Überlebenszeit der Patienten positiv zu beeinflussen [14].

\section{Intraoperativer Ultraschall}

Neben der MRT ist der intraoperative Ultraschall ein wichtiges Instrumentarium zum Auffinden von intraparenchymatösen Prozessen und zur intraoperativen Resektionskontrolle. Die Technologie des Ultraschalls hat in den letzten Jahren ein hohes Maß der Auflösungsfähigkeit erlangt. Mit den neuesten Geräten ist es bereits möglich, 3-D- bzw. 4-D-Ultraschallbilder zu erhalten.

\section{Zukünftige Konzepte}

Intraoperative Magnetresonanztomographie mit

\section{1,5-Tesla- bzw. 3-Tesla-Geräten}

Die nächsten Schritte in der intraoperativen Magnetresonanztomographie beziehen sich auf die Einführung von HochfeldGeräten. Durch 1,5- oder 3-Tesla-Geräte kann eine wesentlich bessere Auflösung erzielt werden. Außerdem ist es denkbar, funktionelle Untersuchung in den operativen Ablauf als Real-time-Untersuchung einzubinden. Neben der verbesserten Auflösung muss jedoch auch mit vermehrten Artefakten gerechnet werden. Eine intraoperative Funktionsdiagnostik ist unter Umständen mit dem Nachteil verbunden, die Patienten in Lokalanästhesie operieren zu müssen. Zukünftige Untersuchungen werden zeigen müssen, inwieweit HochfeldGeräte einen Vorteil hinsichtlich des funktionellen Operierens zeitigen können und ob das Ausmaß der Tumorresektionskontrolle verbessert werden kann. Zur Zeit wird in einigen Zentren Deutschlands ein von der DFG gefördertes Projekt durchgeführt, das den Einsatz von 3-Tesla-Geräten zur Beantwortung von klinischen Fragestellungen beurteilen soll.

\section{Ultraschalldiagnostik}

Die Installation eines intraoperativen MRTs ist äußerst kostenaufwendig und wird nicht in allen Kliniken möglich sein. Hinzu kommt der zeitliche Aufwand, der mit intraoperativen MRT-Kontrollen verbunden ist. Ein Alternativverfahren könnte der intraoperative Ultraschall sein. Die Entwicklungen der letzten Jahre haben gezeigt, dass das Auflösungsvermögen des Ultraschalls erheblich verbessert werden konnte. Außerdem kann durch 3D- und 4D-Untersuchungen ein Tumor im Ultraschall räumlich dargestellt werden. Ein großes Problem besteht in der Unterscheidung von niedergradigem Gliom und Resttumor oder Hirnödem. Durch Einsatz von neuartigen Kontrastmitteln hofft man, dieses Problem zu lösen. Ob das Auflösungsvermögen des Ultraschalls soweit vorangetrieben werden kann, dass dies einer Magnetreso- 
nanztomographie vergleichbar erscheint, werden zukünftige klinische Evaluierungen zeigen. Intraoperativ ist die leichte Handhabung ein deutlicher Vorteil. Der Brain shift spielt auf Grund der Real-time-Information durch den Ultraschall keine Rolle mehr. Von einer norwegischen Arbeitsgruppe [15] wurden bereits Ultraschallbilder von hoher Qualität geliefert, die hoffen lassen, mit dieser Technologie eine zuverlässige Resektionskontrolle und Lokalisationsdiagnostik erreichen $\mathrm{zu}$ können.

\section{Fluoreszenzgestütze Tumordetektion}

Von der Neurochirurgischen Klinik München Großhadern wurde unter Leitung von Professor H.J. Reulen eine Multicenterstudie initiiert, bei der über die orale Verabreichung von 5-Aminolävulinsäure eine fluoreszenzgestützte Tumorexstirpation beim Glioblastom getestet wird. Das Prinzip der Methode beruht auf der intrazellulären enzymatischen Umsetzung der 5-Aminolävulinsäure, die Bestandteil der HämBiosynthese ist. Es konnte nachgewiesen werden, dass in der Tumorzelle ein Mangel an Ferrochelatase besteht. Damit wird die Umsetzung von Protoporphyrin IX, das fluoresziert, blockiert. Die Anfärbung der Tumorzellen ist somit selektiv und beruht nicht auf einer Blut-Hirnschranken-Störung [16]. Die zur Zeit laufende Phase-III-Studie soll klären, ob durch die Anfärbung der Tumorzellen die Radikalität der Tumorexstir- pation verbessert werden kann und sich dies auf die Überlebenszeit der Patienten positiv auswirkt. Die routinemäßige Anfärbung von Tumorzellen könnte eine erhebliche Erleichterung der Tumorexstirpation darstellen.

\section{Ausblick}

Es ist sicher, dass operativ auf Grund der weitreichenden Infiltrationsneigung eine kurative Gliomexstirpation nicht möglich sein wird. Die diffuse Infiltration von Tumorzellen in das Normalgewebe erfordert biologische Therapieansätze. Die bis heute verwirklichten und für die Zukunft geplanten operativen Techniken haben den Funktionserhalt und damit eine Erhöhung der Lebensqualität zum maßgeblichen Ziel. Die Lebensqualität des Patienten ist ein wesentlicher Parameter, der Prognose und Überlebenszeit signifikant positiv beeinflusst. Die Funktionserhaltung wird jedoch immer im reziproken Verhältnis zur Radikalität der Tumorexstirpation stehen. Zukünftige Therapiekonzepte werden, basierend auf einer maximalen Tumorresektion bei Erhaltung der Hirnfunktionen, z.B. systemische oder lokale gentherapeutische Therapiestrategien zum Ziel haben, die über eine Immunmodulation, eine Antiangiogenese, eine Suizidgentherapie oder kombinierte Behandlungsansätze denkbar sind.

\section{Literatur}

1 Burger PC, Heinz ER, Shibata T, Kleihues P: Topographic anatomy and CT correlations in the untreated glioblastoma multiforme. J Neurosurg 1988; 68:698-704

2 Dorward NL, Alberti O, Velani B: Postimaging brain distortion: Magnitude, correlates, and impact on neuronavigation. J Neurosurg 1998;88:656-662.

3 Ojemann GA, Ojemann J, Lettich E, Berger M: Cortical language localization in left, dominant hemisphere. An electrical stimulation mapping investigation in 117 patients. J Neurosurg 1989;71: 316-326.

4 Ojemann GA: Functional mapping of cortical language areas in adults. Intraoperative approaches. Adv Neurol 1993;63:155-163.

5 Herholz K, Reulen HJ, von Stockhausen HM, Thiel A, Ilmberger J, Kessler J, Eisner W, Yoursry TA, Heiss WD: Preoperative activation and intraoperative stimulation of language-related areas in patients with glioma. Neurosurgery 1997;41:12531260.

6 Cedzich C, Taniguchi M, Schäfer S, Schramm J: Somatosensory evoked potential phase reversal and direct motor cortex stimulation during surgery in and around the central region. Neurosurgery 1996; 38:962-970.
7 Coenen VA, Krings T, Mayfrank L, Polin RS, Reinges MH, Thron A, Gilsbach JM: Three-dimensional visualization of the pyramidal tract in a neuronavigation system during brain tumor surgery: First experiences and technical note. Neurosurgery 2001;49:86-92.

8 Duffau H, Capelle L, Lopes M, Faillot T, Sichez JP, Fohanno D: The insular lobe: Physiopathological and surgical considerations. Neurosurgery 2000;47: 801-811.

9 Kaus M, Steinmeier R, Sporer T, Ganslandt O Fahlbusch R: Technical accuracy of a neuronavigation system measured with a high precision mechanical micromanipulator. Neurosurgery 1997;41 1431-1437.

10 Steinmeier R,Rachinger J, Kaus M, Ganslandt O, Huk W, Fahlbusch R: Factors influencing the application accuracy of neuronavigation systems. Stereotact Func Neurosurg 2002; in press.

12 Kreth FW, Berlis A, Spiropoulou V, Faist M, Scheremet R, Rossner R, Volk B, Ostertag CB: The role of tumor resection in the treatment of glioblastoma multiforme in adults. Cancer 1999;86:21172123.

11 Tronnier VM, Wirtz CR, Knauth M, Lenz G, Pastyr O, Bonsanto MM, Albert FK, Kuth R, Staubert A, Schlegel W, Sartor K, Kunze S: Intraoperative diagnostic and interventional magnetic resonance imaging in neurosurgery. Neurosurgery 1997;40:891-900.
12 Steinmeier R, Fahlbusch R, Ganslandt O, Nimsky C, Buchfelder M, Kaus M, Heigl T, Lenz G, Kuth R, Huk W: Intraoperative MRI with the magnetom open: Concepts, neurosurgical indications and procedures. A preliminary report. Neurosurgery 1998; 43:739-748.

13 Black P, Moriarty T, Alexander E 3rd, Stieg P, Woodard EJ, Gleason L, Martin CH, Kikinis R, Schwartz RB, Jolesz FA: Development and implementation of intraoperative magnetic resonance imaging and its neurosurgical applications. Neurosurgery 1997;41:831-8426.

14 Albert FK, Forsting M, Sartor K, Adams,HP, Kunze $\mathrm{S}$ : Early postoperative magnetic resonance imaging after resection of malignant glioma: Objective evaluation of residual tumor and its influence on regrowth and prognosis. Neurosurgery 1994;34:45-60.

15 Gronningsaeter A, Kleven A, Ommedal S, Aarseth TE, Lie T, Lindseth F, Lango T, Unsgard G: SonoWand, an ultrasound-based neuronavigation system. Neurosurgery 2000;47:1373-1379.

16 Stummer W, Stocker S, Wagner S, Stepp H, Fritsch C, Goetz C, Goetz AE, Keifmann R, Reulen HJ: Intraoperative detection of malignant glioma by 5ALA-induced porphyrin fluorescence. Neurosurgery 1998;42;518-526. 